\title{
CASE REPORT: DESMOID TUMOR OF THE BREAST
}

Luciana Carvalho Horta', Fabia Beraldo Silveira², Bruno Henrique Alvarenga1, Anna Carolina Pereira Jácome, Juliana Cristina de Oliveira Lima ${ }^{1}$

${ }^{1}$ Hospital Felicio Rocho - Belo Horizonte (MG), Brazil.

${ }^{2}$ Hospital Felicio Rocho - Belo Horizonte (MG), Brazil.

Desmoid tumors are neoplasms that originate from fascial or musculoaponeurotic structures, constituted by fibroblast proliferation. It has no metastatic or differentiation potential, but is locally aggressive and has high rates of recurrence even after complete resection. It represents $0.03 \%$ of all neoplasms. This study aimed at reporting a rare clinical case of a young patient diagnosed with a desmoid tumor of the breast after a silicone implant surgery. 